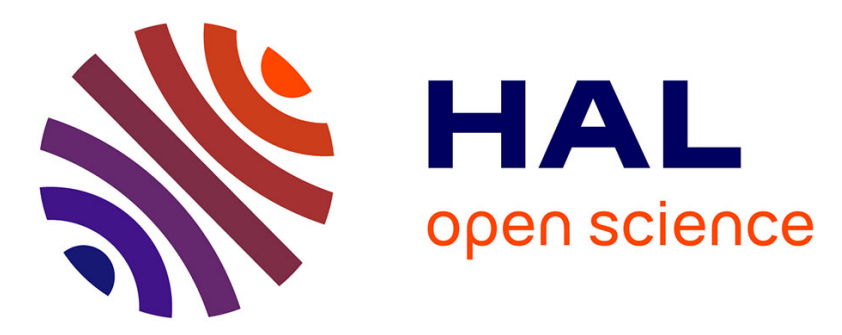

\title{
Description d'un appareillage de mesure d'aimantation sous champs forts
}

\author{
J.L. Féron, G. Hug, P Morin, J.C. Picoche, J.P. Rebouillat
}

\section{To cite this version:}

J.L. Féron, G. Hug, P Morin, J.C. Picoche, J.P. Rebouillat. Description d'un appareillage de mesure d'aimantation sous champs forts. Revue de Physique Appliquée, 1971, 6 (1), pp.55-58. 10.1051/rphysap:019710060105500 . jpa-00243502

\section{HAL Id: jpa-00243502 https://hal.science/jpa-00243502}

Submitted on 1 Jan 1971

HAL is a multi-disciplinary open access archive for the deposit and dissemination of scientific research documents, whether they are published or not. The documents may come from teaching and research institutions in France or abroad, or from public or private research centers.
L'archive ouverte pluridisciplinaire HAL, est destinée au dépôt et à la diffusion de documents scientifiques de niveau recherche, publiés ou non, émanant des établissements d'enseignement et de recherche français ou étrangers, des laboratoires publics ou privés. 


\title{
DESCRIPTION D'UN APPAREILLAGE DE MESURE D'AIMANTATION SOUS CHAMPS FORTS
}

\author{
J. L. FÉRON, G. HUG, P. MORIN, J. C. PICOCHE et J. P. REBOUILlAT \\ Laboratoire d'Electrostatique et de Physique du Métal \\ C. N. R. S., Cédex 166, 38, Grenoble \\ (Reçu le 21 octobre 1970)
}

\begin{abstract}
Résumé. - Nous décrivons un ensemble expérimental de mesure d'aimantations dans des champs atteignant $150 \mathrm{kOe}$ créés par des bobines à eau. Le domaine de température s'étend de 1,4 à $300^{\circ} \mathrm{K}$. Nous discutons des avantages respectifs de deux magnétomètres, l'un analogique, l'autre numérique. Les fluctuations du champ constituent la limite essentielle de la précision et de la sensibilité de l'appareillage.
\end{abstract}

Abstract. - An experimental apparatus of magnetization measurements is described. High magnetic fields up to $150 \mathrm{kOe}$ are generated by water-cooled magnets. The temperature of the sample varies from 1.2 to $300 \mathrm{~K}$. We discuss respective advantages of two magnetometers, one analogical, the other one digital. The magnetic field fluctuations are the main limit of accuracy and sensitivity of the apparatus.

Introduction. - Dans la plupart des problèmes de magnétisme, il est intéressant de connaître l'aimantation des substances soumises à des champs magnétiques forts. Nous avons réalisé, dans le cadre du Laboratoire de Champs Forts du L. E. P. M. [1], un appareillage de mesure d'aimantations utilisant la méthode de flux. Cette expérience permet des mesures précises avec une large gamme de sensibilités dans des champs magnétiques atteignant $150 \mathrm{kOe}$ et à des températures comprises entre 1,2 et $300^{\circ} \mathrm{K}$. De nombreux problèmes ont dû être résolus : création d'un champ magnétique stable, régulation de la température de l'échantillon, réalisation de fluxmètres précis et sensibles, étalonnages et automatisation des mesures.

A. Champs magnétiques forts. - Les champs magnétiques forts sont créés par des bobines à eau. Pour obtenir des champs supérieurs à $80 \mathrm{kOe}$ dans un diamètre utile de $50 \mathrm{~mm}$, il faut une puissance électrique de $1700 \mathrm{k} \mathrm{W}$ [2].

A.1 Production DU CHAMP. - L'alimentation électrique est constituée d'une génératrice à courant continu, fournissant, en régime nominal, une intensité de $5000 \mathrm{~A}$. La puissance est régulée par un système à deux boucles superposées. Aux basses fréquences, des essais effectués à l'aide d'un enregistreur galvanométrique indiquent une stabilité du courant de $\pm 5 \times 10^{-5}$ pour toute valeur du courant. L'analyse à fréquences supérieures révèle l'existence de bruits intrinsèques à la génératrice à $12,5,50,100$ et $1650 \mathrm{~Hz}$. Les fluctuations résultantes du courant restent inférieures à $10^{-4}$.

Le refroidissement des bobines est assuré par de l'eau très pure circulant dans un circuit fermé en cuivre et en acier inoxydable. La capacité calorifique de l'eau en fait le meilleur véhicule de puissances thermiques importantes. Après déminéralisation, sa résistivité est suffisamment forte, $2 \mathrm{M} \Omega . \mathrm{cm}$ pour minimiser les risques d'amorçage à l'intérieur des bobines.

Deux types de bobines sont utilisées dans le laboratoire : les bobines à densité de courant constante [3] comprennent les galettes formées par l'enroulement en spirale d'un méplat de cuivre, en contact avec l'eau de refroidissement (Fig. 1). Pour un diamètre intérieur de $51 \mathrm{~mm}$, le champ a une valeur maximale de $86 \mathrm{kOe}$;

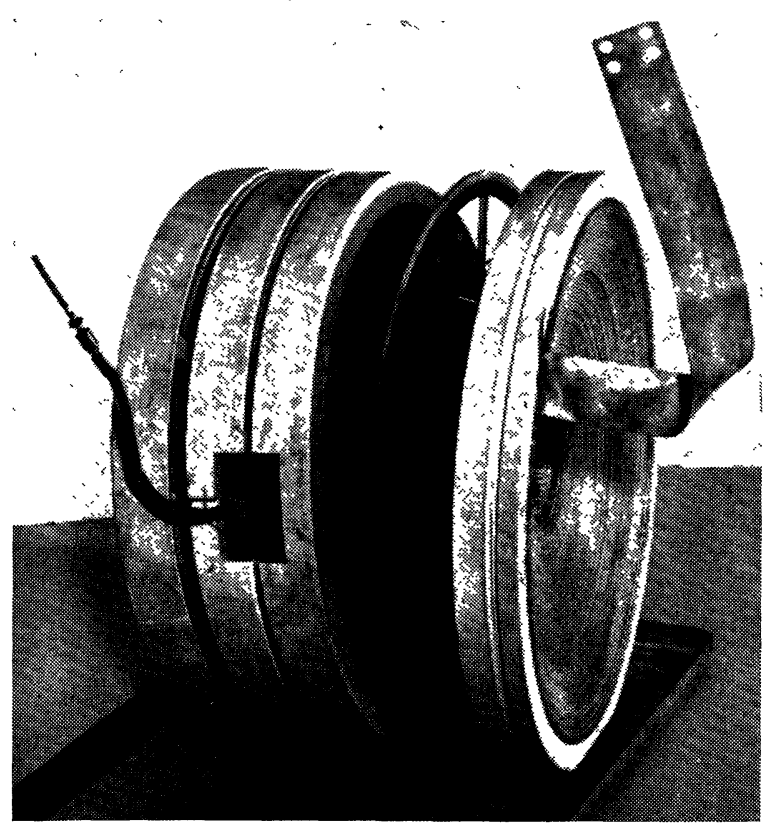

FIG. 1. - Bobinage en galettes du type Wood. 
son homogénéité axiale $\left(H-H_{\max }\right) / H_{\max }$ au centre de la bobine, est inférieure à $1 \%$ sur une distance de $24 \mathrm{~mm}$. Les bobines de Bitter à densité de courant variable sont constituées par un empilement de disques, de faible épaisseur (Fig. 2). La surface d'échange ther-

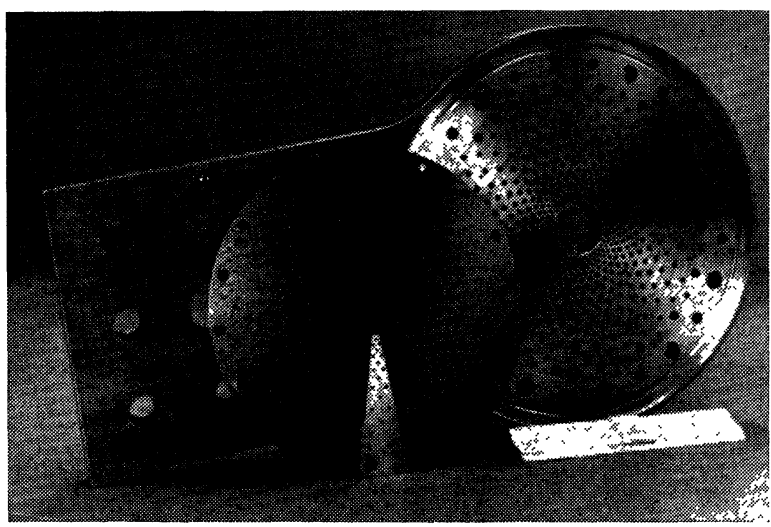

FIG. 2. - Bobine de Bitter de 110 kOe : Plateau d'amenée de courant et disque de Bitter.

mique de ces bobines étant très faible, le refroidissement hydraulique doit en être optimisé $[1,4]$. Selon le diamètre intérieur, 30 ou $15 \mathrm{~mm}$, le champ au centre de la bobine de Bitter pour la puissance maximale est de $110 \mathrm{kOe}$ ou $140 \mathrm{kOe}$.

A.2 Etalonnage DU CHAMP. - Une résistance $100 \mu \Omega$, en manganine, refroidie par circulation d'eau, sert simultanément à la régulation et à la mesure du courant de la génératrice. La mesure de la tension à ses bornes se fait en utilisant un pont potentiométrique ou un voltmètre numérique de forte impédance. Le champ maximal au centre des bobines est proportionnel à la variation de flux dans un petit enroulement lors de son déplacement du centre à une région où le champ est nul. Cette variation de flux est mesurée à l'aide d'un intégrateur numérique ; la surface du petit enroulement est déterminée de façon similaire dans un champ référence connu par résonance magnétique nucléaire. Dans les différentes bobines utilisées, nous avons vérifié que la correspondance entre le courant inducteur et le champ induit est linéaire. La valeur du champ est obtenue par la mesure du courant. Les fluctuations du champ proviennent d'une part des fluctuations de courant, d'autre part des instabilités mécaniques internes des bobines, dues aux conditions extrêmes de refroidissement et de contraintes; elles limitent la précision sur la mesure du champ à $2 \times 10^{-4}$.

B. Température et aimantation. - Nous décrivons le système de régulation thermique, et ensuite le système de mesures d'aimantations.

B.1 Mesure et RÉGUlation DE TEMPÉrature. Nous utilisons un vase cryogénique à hélium liquide de grande autonomie. Un calorimètre sert à isoler l'échantillon du bain, un enroulement chauffant permet d'atteindre la température désirée. L'échantillon de diamètre $6 \mathrm{~mm}$ ou $3 \mathrm{~mm}$ selon les bobines de puissance utilisées est en contact thermique étroit avec deux thermosondes, l'une en platine, l'autre en carbone. Elles sont insérées dans un montage en pont, l'équilibre étant détecté par un enregistreur galvanométrique Graphispot. La sonde de platine, Lyon-Alemand, a une sensibilité moyenne entre 40 et $300^{\circ} \mathrm{K}$ de $210 \mathrm{~m} \Omega /{ }^{\circ} \mathrm{K}$; nous l'étalonnons avec précision à l'aide des points fixes habituels et des tables du National Bureau of Standards [5]. Au-dessous de $30^{\circ} \mathrm{K}$, nous utilisons une sonde de carbone 1/10 watt Allen-Bradley.

La température est stabilisée à l'aide d'une régulation de type proportionnel et intégral. Le premier amplificateur d'erreur est constitué par le potentiomètre reproducteur du Graphispot. Ceci permet d'utiliser la thermosonde comme organe détecteur du signal d'erreur sans pour cela perturber la mesure de la température.

B. 2 Mesure de L'Aimantation. - Nous utilisons la méthode d'extraction proposée en 1905 par P. Weiss [6] : l'échantillon, soumis à un champ magnétique constant, est placé entre deux bobines de détection connectées en série-opposition; la variation de flux ainsi engendrée est proportionnelle à l'aimantation de l'échantillon. Cependant, les possibilités de l'appareillage sont limitées par le bruit de fond créé par les fluctuations du champ magnétique. Afin de le réduire, nous avons amélioré le dispositif des bobines de détection : ces bobines sont placées à l'intérieur du vase cryogénique ce qui augmente leur couplage avec l'échantillon et diminue les forces électromotrices parasites induites. Un couple de bobines de compensation est disposé concentriquement aux bobines de détection et connecté en série-opposition avec cellesci ; il minimise les fluctuations parasites du champ d'ordre mécanique.

Nous disposons, de deux méthodes de mesure: l'une analogique, l'autre numérique.

- Dans le premier système de mesure, le champ et la température sont déterminés par une méthode d'opposition; la variation de flux proportionnelle à l'aimantation est intégrée par un galvanomètre balistique. L'utilisation d'un suiveur de spot pour enregistrer les déviations du galvanomètre permet d'améliorer la résolution de l'appareillage par rapport à une lecture visuelle. Cette méthode est manuelle et n'est pas susceptible d'être automatisée.

- La méthode de mesure numérique permet d'assurer l'automatisation de l'expérience, d'où une grande rapidité de travail et une facilité accrue dans le dépouillement des résultats. La mesure des paramètres, champ magnétique et température de l'échantillon, peut être effectuée à l'aide d'un voltmètre numérique standard. En revanche, la mesure de 
l'aimantation n'est possible, dans la méthode adoptée, qu'avec un fluxmètre. Les seuls fluxmètres digitaux existants à temps d'intégration variable utilisent le principe de la conversion tension-fréquence [7]. Nous avons employé un voltmètre numérique intégrateur (I. D. V. M.), dont la résolution est de 200 Maxwell par digit. Pour nos bobines de mesure, la variation de flux correspondant à une aimantation de 1 u. e. m. est de 60000 Maxwell ; la résolution est donc de $3,3 \times 10^{-3}$ u.e.m./digit.

Un centralisateur de mesure a été réalisé à partir de ce voltmètre. Un commutateur de voies permet de mesurer successivement tous les paramètres; les résultats sont simultanément imprimés et perforés sur ruban en vue d'un traitement par ordinateur. D'autre part, un convertisseur numérique analogique relié à un enregistreur XY permet de tracer point par point la variation d'un paramètre en fonction d'un autre. L'automatisation de l'expérience est obtenue grâce à un programmateur qui synchronise les différentes mesures, le mouvement de l'échantillon, la variation du champ magnétique (Fig. 3).

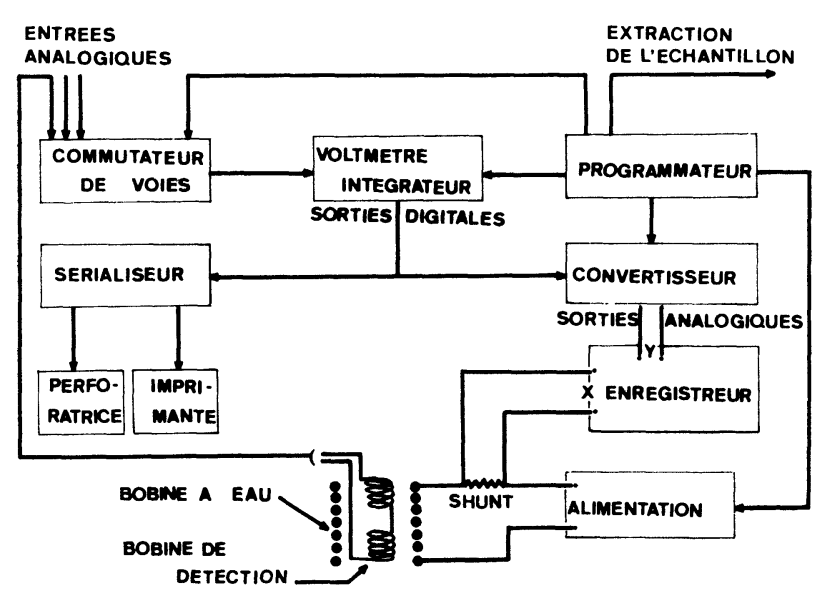

Fig. 3. - Schéma de principe du système de mesure analogique.

B. 3 Performances. - Pour une courbe d'aimantation isotherme, la température de la thermosonde est maintenue étroitement autour de la température désirée : ses variations sont toujours inférieures à $\pm 0,05^{\circ} \mathrm{K}$. La thermosonde est en contact avec l'échantillon, elle est donc soumise au champ magnétique maximum. Il faut tenir compte des corrections de magnétorésistance, qui ont été établies par interpolations linéaires entre leurs valeurs mesurées à $77^{\circ} \mathrm{K}$ et $300^{\circ} \mathrm{K}$. Négligée, la magnétorésistance entraînerait pour un champ de $86 \mathrm{kOe}$, une erreur systématique de $0,4^{\circ} \mathrm{K}$ à $77^{\circ} \mathrm{K}$ et de $0,1^{\circ} \mathrm{K}$ à $300^{\circ} \mathrm{K}$.

La méthode de mesure utilisant le galvanomètre permet la détermination de faibles aimantations; les réglages de sensibilité en sont aisés. Malheureusement, la précision et la reproductibilité des mesures sont limitées. L'erreur relative sur l'aimantation $\Delta M / M$ est $5 \times 10^{-3}$ : elle est constante pour toutes les sensibilités, sauf pour les petites aimantations, en raison du bruit de fond. Une susceptibilité de $3 \times 10^{-5}$ u. e. m. est alors entachée d'une erreur relative constante atteignant $3 \%$. Cet appareillage est d'une grande robustesse, il est opérationnel depuis 1965.

La méthode numérique couvre la même gamme d'aimantation que la méthode précédente. En revanche, sa précision et sa reproductibilité sont très nettement supérieures; si la méthode galvanométrique permet des mesures de l'aimantation entachées d'une erreur relative constante, la méthode numérique conduit à une erreur absolue constante sur l'aimantation, $\Delta M: \Delta M$ reste de l'ordre de $10^{-2}$ u. e. $m$. pour toutes les valeurs mesurables de l'aimantation. En outre, le voltmètre numérique permet une détermination très précise des paramètres, champ et température.

Afin de profiter au maximum des performances du centralisateur de mesure, des réglages beaucoup plus précis sont nécessaires : position mécanique de l'échantillon en fonction de la température, correction de porte-échantillon systématique en fonction du champ, stabilité de la température d'une courbe d'aimantation isotherme.

Conclusion. - Dans les bobines à eau, les fluctuations du champ magnétique dans l'espace et dans le temps sont relativement importantes. Il en résulte que la sensibilité et la précision de tout système de mesure d'aimantation sont limitées par la présence de flux parasites induits aux bornes des bobines de détection. Par l'optimisation et la compensation de ces bobines, nous avons réduit les flux parasites à leur valeur minima, qui, quel que soit le type de fluxmètre utilisé, impose une erreur absolue sur l'aimantation de $10^{-2}$ u.e. $\mathrm{m}$. Cet ensemble expérimental a permis l'étude de substances faiblement magnétiques avec une résolution satisfaisante [8,9]. Dans le cas de fortes aimantations $[10,11]$, l'usage du fluxmètre numérique a considérablement amélioré la précision qui peut alors atteindre $2 \times 10^{-4}$. Par ailleurs, l'automatisation de l'appareillage permet de multiplier les points d'une courbe d'aimantation isotherme et d'en améliorer ainsi la définition.

Une amélioration sensible des performances du magnétomètre pourrait être obtenue par une meilleure régulation du courant inducteur et une plus grande homogénéité axiale du champ. Cette dernière modification ne peut se faire qu'au détriment, pour une puissance électrique installée, de la valeur maximale du champ.

Nous remercions le Professeur R. Pauthenet qui a favorisé la réalisation de ce travail. 


\section{Bibliographie}

[1] Boissier (R.), Brichant (F.), Goyer (J.), Fournier (J.), Ricque (R.), Feron (J. L.), Pauthenet (R.), PICOCHE (J. C.), Les champs magnétiques intenses, leur production et leurs applications, Editions du C. N. R. S., 1967, 83.

[2] Picoche (J. C.), Thèse de Docteur-Ingénieur, Université de Grenoble, 1969.

[3] Daniels (J. M.), Proc. Phys. Soc., 1953, B 66, 921.

[4] Fournier (J.), Thèse de Docteur-Ingénieur, Université de Grenoble, 1966.

[5] Stimson (H. F.), Journal of Research of the N. B. S., 1961, 65A, 139.
[6] Weiss (P.), J. Phys. Rad., 1905, 4, 473.

[7] Rebouillat (J. P.), A paraître.

[8] Bloch (D.), Feron (J. L.), Georges (R.), Jacobs (I. S.), J. Appl. Phys., 1967, 38, 1474.

[9] Feron (J. L.), Hug (G.), Pauthenet (R.), Les éléments des terres rares. Editions du C. N. R.S., 1970, 2, 19.

[10] Boutron (P.), Feron (J. L.), Hug (G.), Morin (P.), Conférence Internationale de Magnétisme, 1970. $J$. Physique (à paraître).

[11] Hug (G.), MoRIN (P.), Conférence Internationale de Magnétisme, 1970. J. Physique (à paraître). 